\title{
Conditions for large earthquakes in a two-asperity fault model
}

\author{
M. Dragoni ${ }^{1}$ and S. Santini ${ }^{2}$ \\ ${ }^{1}$ Dipartimento di Fisica, Università di Bologna, Viale Carlo Berti Pichat 8, 40127 Bologna, Italy \\ ${ }^{2}$ Dipartimento di Scienze di Base e Fondamenti, Università di Urbino, Via Santa Chiara 27, 61029 Urbino, Italy
}

Received: 30 June 2011 - Revised: 23 August 2011 - Accepted: 11 October 2011 - Published: 17 October 2011

\begin{abstract}
A fault with two asperities is modelled as a system made of two blocks coupled by a spring and sliding on a plane under the same values of static and dynamic friction. An analytical solution is given for the simultaneous motion of the blocks and the corresponding orbits are plotted in the phase space. It is proven that, whichever the initial state is, the long-term behaviour of the system is one of an infinite number of limit cycles, characterized by a particular pattern of forces. The region where the system is located when the blocks are stationary can be divided into narrow stripes corresponding to different orbits of the points belonging to them. This implies that the system is sensitive to perturbations and has relevant implications for a fault, which is subject to stress transfers from earthquakes generated by neighbouring faults. In this case, the fault may experience a larger earthquake, with the simultaneous failure of the two asperities, which restores a stress distribution compatible with periodic behaviour. The seismic moment associated with simultaneous asperity failure is always greater than the maximum value that can be released in a limit cycle. For strongly coupled asperities, the moment can be several times larger.
\end{abstract}

\section{Introduction}

The long-term behaviour of seismic sources can be usefully studied by means of dynamical models with a finite number of degrees of freedom. Such models include the essential features of seismic sources, i.e. a stick-slip mechanism activated by applied forces, but avoid the detailed field description of continuum mechanics. The advantage is that we can follow the evolution of the system in the phase space and obtain a

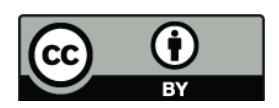

Correspondence to: S. Santini (santini@fis.uniurb.it) deeper understanding of the long-term behaviour of the system itself.

Low-order analogs of seismic sources can be represented as spring-block systems and were first introduced by Burridge and Knopoff (1967). Different numbers of blocks and friction laws have been considered by following authors (Byerlee, 1978; Dieterich, 1981; Ruina, 1983; Rice and Tse, 1986; Gu and Wong, 1991; Belardinelli and Belardinelli, 1996; Erickson et al., 2008) showing that spring-block models can simulate several features of seismic activity (Dieterich, 1972; Rundle and Jackson, 1977; Cohen, 1977; Cao and Aki, 1984, 1986; Gu et al., 1984; Carlson and Langer, 1989a,b; Huang and Turcotte, 1990b; Carlson et al., 1994). Spring-block systems are nonlinear, dissipative, piecewisesmooth dynamical systems (di Bernardo et al., 2008).

The simplest fault system is made of two distinct fault segments or of two asperities on the same fault and can be simulated by two coupled blocks pulled at constant velocity. Two-block systems were considered by Nussbaum and Ruina (1987); Huang and Turcotte (1990a, 1992); McCloskey and Bean (1992); de Sousa Vieira (1995); He (2003). Galvanetto (2004) studied a similar system made of two blocks connected by a spring and placed on a moving belt.

Turcotte (1997) showed numerically that a symmetric twoblock system can exhibit limit cycles in the phase space, representing the alternate motion of the blocks. Dragoni and Santini (2010) solved analytically the equations for the motion of one block at a time, showing that there exists a region $L$ in the phase space, from which the system enters immediately a limit cycle. There is an infinite number of such cycles, the shapes of which are determined by the position of the representative point in $L$. Such a position is related to the inhomogeneity of the stress applied to the fault.

The present paper completes the study of Dragoni and Santini (2010) by considering the evolution of the system when the representative point is outside $L$ and showing that in this case the motion of one block can trigger the motion

Published by Copernicus Publications on behalf of the European Geosciences Union and the American Geophysical Union. 


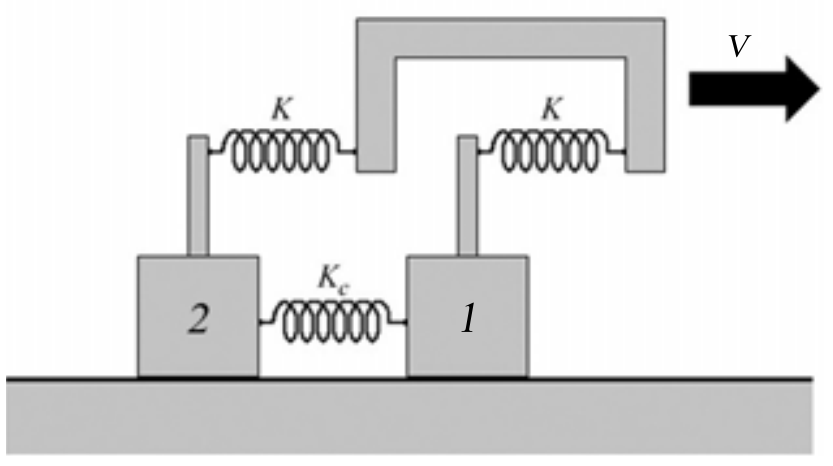

Fig. 1. The two-block system.

of the other. We present an analytical solution for the simultaneous motion of the blocks and show that it represents the occurrence of the largest earthquakes that can be produced by the fault.

\section{The model}

We model the fault as a system made of two blocks having equal mass $m$ and placed on a horizontal plane (Fig. 1). Each block is connected by a horizontal spring of rigidity $K$ to a driving mechanism moving at constant velocity $v$ in the horizontal direction. The blocks are connected to each other by a spring of rigidity $K_{c}$. We assume that the motion of each block is resisted by a static friction $f_{\mathrm{s}}$ and a dynamic friction $f_{\mathrm{d}}$. We define

$\epsilon=\frac{f_{\mathrm{d}}}{f_{\mathrm{s}}}, \quad \alpha=\frac{K_{c}}{K}$

where $0<\epsilon<1$ and $\alpha>0$. It is shown below that the seismological application of the model requires $\epsilon \geq 1 / 2$.

Let $x(t)$ and $y(t)$ be the extensions of the springs connecting respectively blocks 1 and 2 to the driver as functions of time $t$. We introduce the nondimensional quantities

$X=\frac{K x}{f_{\mathrm{s}}}, \quad Y=\frac{K y}{f_{\mathrm{s}}}, \quad T=\sqrt{\frac{K}{m}} t, \quad V=\frac{\sqrt{K m}}{f_{\mathrm{s}}} v$

If $f_{1}$ and $f_{2}$ are the forces applied to blocks 1 and 2 respectively, we define nondimensional forces

$F_{1}=\frac{f_{1}}{f_{\mathrm{s}}}, \quad F_{2}=\frac{f_{2}}{f_{\mathrm{s}}}$

In nondimensional form, the equations of motion of the system are

$\ddot{X}=0, \quad \ddot{Y}=0$

when the blocks are stationary and

$\ddot{X}+(1+\alpha) X=\epsilon+\alpha Y$
$\ddot{Y}+(1+\alpha) Y=\epsilon+\alpha X$

when the blocks are moving. Here dots indicate differentiation with respect to $T$.

We study the evolution of the system in the phase space and precisely on the plane $X Y$, where the representative point of the system is located for most time. The projection of the phase space on this plane is the unit square with vertices at $(0,0),(1,0),(1,1),(0,1)$. The conditions for the onset of motion of blocks 1 and 2 are represented respectively by lines

$Y=\frac{1+\alpha}{\alpha} X-\frac{1}{\alpha}$

$Y=\frac{\alpha}{1+\alpha} X+\frac{1}{1+\alpha}$

that we call line 1 and line 2. Accordingly, the points corresponding to stationary blocks belong to a quadrilateral $Q$ with vertices at points $(0,0),(A, 0),(1,1),(0, A)$, where

$A=\frac{1}{1+\alpha}$

As initial point of the orbit, we choose a point $P_{0}=$ $\left(X_{0}, Y_{0}\right) \in Q$. When the blocks are stationary, the orbit is a segment of line

$Y=X+p$

where

$p=Y_{0}-X_{0}, \quad-A \leq p \leq A$

In Dragoni and Santini (2010) we defined the sets

$L_{1}=\left\{P_{0} \in Q ;-b<p<-a\right\}$

$L_{2}=\left\{P_{0} \in Q ; a<p<b\right\}$

where

$a=\frac{\alpha}{1+2 \alpha} U, \quad b=\frac{1+\alpha}{1+2 \alpha} U, \quad U=2 \frac{1-\epsilon}{1+\alpha}$

with $a+b=U$. If $P_{0}$ belongs to $L=L_{1} \cup L_{2}$, the system is in a limit cycle or enters it immediately. Such cycles are characterized by the alternate motion of the blocks with an amount $U$ of slip. In the next section we consider the general solution of the equations of motion, including the case when the blocks move simultaneously.

Observations show that the direction of tectonic stress applied to a fault is stable: following fault slip, a decrease in shear stress is observed, but not an inversion of its direction. The seismological application of the model requires then $F_{1} \leq 0$ and $F_{2} \leq 0$, or

$X \geq 0, \quad Y \geq 0$

At the beginning of a slip event, the representative point of the system belongs to line 1 or 2 . For points belonging to such lines, the smallest value of $X$ and $Y$ respectively is $A$. Conditions (15) are then satisfied if $A-U \geq 0$, whence $\epsilon \geq$ $1 / 2$ from Eqs. (9) and (14). 


\section{Motion of a single block}

A block can move from a stationary state or after a segment in which it moves simultaneously to the other block. The solution for the former case was given in Dragoni and Santini (2010). More generally, for the motion of block 1 we assume as initial conditions

$X(0)=\bar{X}, \quad \dot{X}(0)=\bar{V}$

During the motion of block $1, Y$ has a constant value $\bar{Y}$. Hence the motion is solution of Eq. (5) with $Y=\bar{Y}$ :

$X(T)=\frac{\epsilon+\alpha \bar{Y}}{\omega^{2}}+\left(\bar{X}-\frac{\epsilon+\alpha \bar{Y}}{\omega^{2}}\right) \cos \omega T+\frac{\bar{V}}{\omega} \sin \omega T$

where

$\omega=\sqrt{1+\alpha}$

The velocity $\dot{X}(T)$ vanishes when $T$ is equal to

$T_{1}=\frac{1}{\omega} \arctan \frac{\omega \bar{V}}{\omega^{2} \bar{X}-\epsilon-\alpha \bar{Y}}$

The point at which the motion stops is then $X=X\left(T_{1}\right), Y=$ $\bar{Y}$.

The solution for the motion of block 2 is analogous. We assume as initial conditions

$Y(0)=\bar{Y}, \quad \dot{Y}(0)=\bar{V}$

During the motion of block $2, X$ has a constant value $\bar{X}$. Hence the motion is solution of Eq. (6) with $X=\bar{X}$ :

$Y(T)=\frac{\epsilon+\alpha \bar{X}}{\omega^{2}}+\left(\bar{Y}-\frac{\epsilon+\alpha \bar{X}}{\omega^{2}}\right) \cos \omega T+\frac{\bar{V}}{\omega} \sin \omega T$

The velocity $\dot{Y}(T)$ vanishes when $T$ is equal to

$T_{2}=\frac{1}{\omega} \arctan \frac{\omega \bar{V}}{\omega^{2} \bar{Y}-\epsilon-\alpha \bar{X}}$

The point at which the motion stops is then $X=\bar{X}, Y=$ $Y\left(T_{2}\right)$.

\section{Simultaneous block motion}

It may happen that, while a block is moving, the condition for the motion of the other block is attained. In this case we have the simultaneous motion of the two blocks and Eqs. (5) and (6) must be solved simultaneously. Two different initial conditions are possible.

If the motion of block 1 triggers that of block 2, initial conditions are
$X=\bar{X}, \quad Y=\bar{Y}, \quad \dot{X}=\bar{V}, \quad \dot{Y}=0$

where $\bar{X}$ and $\bar{Y}$ are related by Eq. (8). The solution is

$$
\begin{aligned}
X(T)= & \epsilon-\frac{2 \epsilon-\bar{X}-\bar{Y}}{2} \cos T+\frac{\bar{V}}{2} \sin T+\frac{\bar{X}-\bar{Y}}{2} \cos \Omega T \\
& +\frac{\bar{V}}{2 \Omega} \sin \Omega T
\end{aligned}
$$

$$
\begin{aligned}
Y(T)= & \epsilon-\frac{2 \epsilon-\bar{X}-\bar{Y}}{2} \cos T+\frac{\bar{V}}{2} \sin T-\frac{\bar{X}-\bar{Y}}{2} \cos \Omega T \\
& -\frac{\bar{V}}{2 \Omega} \sin \Omega T
\end{aligned}
$$

where

$\Omega=\sqrt{1+2 \alpha}$

In order to find which block stops earlier and at what time, we must solve the equations

$\dot{X}=0, \quad \dot{Y}=0$

giving the trigonometric equations

$$
\begin{aligned}
& (2 \epsilon-\bar{X}-\bar{Y}) \sin T+\bar{V} \cos T-(\bar{X}-\bar{Y}) \Omega \sin \Omega T \\
+ & \bar{V} \cos \Omega T=0 \\
& (2 \epsilon-\bar{X}-\bar{Y}) \sin T+\bar{V} \cos T+(\bar{X}-\bar{Y}) \Omega \sin \Omega T \\
- & \bar{V} \cos \Omega T=0
\end{aligned}
$$

that must be solved numerically. If we call $T_{x}$ and $T_{y}$ the smallest positive solutions of Eqs. (28) and (29) respectively, the duration of simultaneous block motion is

$T_{\mathrm{S}}=\min \left(T_{x}, T_{y}\right)$

The result depends on the value of $p$ : there exists a positive number $c$ such that $T_{\mathrm{S}}=T_{x}$ or $T_{\mathrm{S}}=T_{y}$ according to whether $p<-c$ or $p>-c$ (Appendix A).

If the motion of block 2 triggers that of block 1, initial conditions are

$X=\bar{X}, \quad Y=\bar{Y}, \quad \dot{X}=0, \quad \dot{Y}=\bar{V}$

where $\bar{X}$ and $\bar{Y}$ are related by Eq. (7). The solution is

$$
\begin{aligned}
X(T)= & \epsilon-\frac{2 \epsilon-\bar{X}-\bar{Y}}{2} \cos T+\frac{\bar{V}}{2} \sin T+\frac{\bar{X}-\bar{Y}}{2} \cos \Omega T \\
& -\frac{\bar{V}}{2 \Omega} \sin \Omega T \\
Y(T)= & \epsilon-\frac{2 \epsilon-\bar{X}-\bar{Y}}{2} \cos T+\frac{\bar{V}}{2} \sin T-\frac{\bar{X}-\bar{Y}}{2} \cos \Omega T \\
& +\frac{\bar{V}}{2 \Omega} \sin \Omega T
\end{aligned}
$$

Equations analogous to (28), (29) and (30) must be solved in order to find which block stops earlier and at what time. 


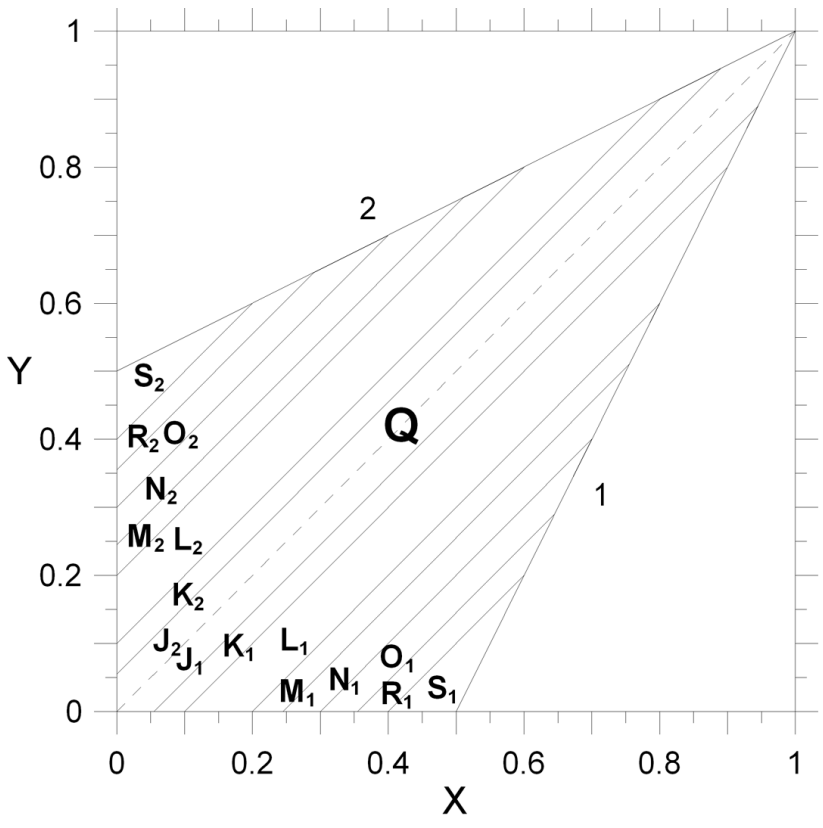

Fig. 2. Projection of the phase space in the plane $X Y$ : the region $Q$ $(\alpha=1)$ and its subsets $(\epsilon=0.7)$.

\section{Orbits in the phase space}

The evolution of the system depends on the position of $P_{0}$. It results that $Q$ can be divided into several subsets corresponding to different orbits of the points belonging to them. These subsets are narrow stripes parallel to the major diagonal of $Q$ (Fig. 2). Thanks to the symmetry of the problem, we may consider only the case $p \leq 0$. Accordingly, the part of $Q$ below the diagonal can be divided into the following subsets:

$$
\begin{aligned}
& J_{1}=\left\{P_{0} \in Q ;-c<p<0\right\} \\
& K_{1}=\left\{P_{0} \in Q ;-a<p<-c\right\} \\
& L_{1}=\left\{P_{0} \in Q ;-b<p<-a\right\} \\
& M_{1}=\left\{P_{0} \in Q ;-U+c<p<-b\right\} \\
& N_{1}=\left\{P_{0} \in Q ;-U<p<-U+c\right\} \\
& O_{1}=\left\{P_{0} \in Q ;-U-c<p<-U\right\} \\
& R_{1}=\left\{P_{0} \in Q ;-U-a<p<-U-c\right\} \\
& S_{1}=\left\{P_{0} \in Q ;-A<p<-U-a\right\}
\end{aligned}
$$

The part above the diagonal can be divided symmetrically into subsets $J_{2}, K_{2}, L_{2}, M_{2}, N_{2}, O_{2}, R_{2}, S_{2}$. We next consider the evolution of the system from $P_{0}$ to another point belonging to $Q$ through a slip event which may involve one or both blocks.
Case $p=0$. In this particular case the coupling spring is undeformed and there is no coupling of the blocks. The orbit begins as a segment of the major diagonal of $Q$. When it reaches the point $P_{1}=(1,1)$, the blocks move together. The initial conditions for block motion are then

$\bar{X}=\bar{Y}=1, \quad \dot{X}=\dot{Y}=0$

From either Eqs. (24)-(25) or (32)-(33) it follows

$X(T)=Y(T)=\epsilon+(1-\epsilon) \cos T$

The solution coincides with that of a single block. The representative point goes along the same line backward until $T=\pi$, when the blocks stop. The coordinates of the arrest point are $X_{2}=Y_{2}=2 \epsilon-1$. The limit cycle is then the line segment with end points $(1,1)$ and $\left(X_{2}, Y_{2}\right)$. The states with $p=0$ are unstable, because an arbitrarily small perturbation will move the representative point of the system to region $J_{1}$ or $J_{2}$, whence the point will follow a completely different orbit. This is particularly true for a fault, which can not be considered an isolated system.

Case $P_{0} \in J_{1}$. The orbit intercepts line 1 at $P_{1}$ and block 1 moves. While block 1 is moving, the orbit intercepts line 2 at $P_{2}$ : the motion of block 1 triggers the motion of block 2 . The orbit leaves $Q$ and becomes curvilinear. For a certain interval of time the blocks move simultaneously, then block 2 stops at $P_{3}$. The coordinates of $P_{3}$ must be calculated numerically (Appendix A). Block 1 continues its motion to point $P_{4}$. For most values of $p, P_{4}$ is in $K_{1}$ (Fig. 3a). Only orbits with $p$ very close to 0 come back to $J_{1}$, but with a smaller value of $p$ (Fig. 3b). From here they reach $K_{1}$.

Case $P_{0} \in K_{1}$. The orbit is similar to that previously described up to point $P_{2}$, when the motion of block 1 triggers the motion of block 2, the orbit leaves $Q$ and becomes curvilinear. However, in the present case block 1 stops earlier than block 2: this occurs at point $P_{3}$. The coordinates of $P_{3}$ must be calculated numerically (Appendix A). Block 2 continues it motion to point $P_{4}$. This point is in $L_{1}$ and the orbit enters a limit cycle (Fig. 3c).

Case $P_{0} \in L_{1}$. It is the case considered in Dragoni and Santini (2010). The orbit enters immediately a limit cycle, the shape of which is determined by the value of $p$.

Case $P_{0} \in M_{1}$. The orbit intercepts line 1 at $P_{1}$ and block 1 moves to point $P_{2}$, which belongs to the region $K_{2}$. Hence the orbit continues as an orbit with $p>0$, that we do not need to consider due to the symmetry.

Case $P_{0} \in N_{1}$. The orbit intercepts line 1 at $P_{1}$ and block 1 moves to point $P_{2}$, which belongs to the region $J_{2}$. Hence the orbit continues as an orbit with $p>0$.

Case $P_{0} \in O_{1}$. The orbit intercepts line 1 at $P_{1}$ and block 1 moves to point $P_{2}$, which belongs to the region $J_{1}$. Hence the orbit continues as described above.

Case $P_{0} \in R_{1}$. The orbit intercepts line 1 at $P_{1}$ and block 1 moves to point $P_{2}$, which belongs to the region $K_{1}$. Hence the orbit continues as described above. 

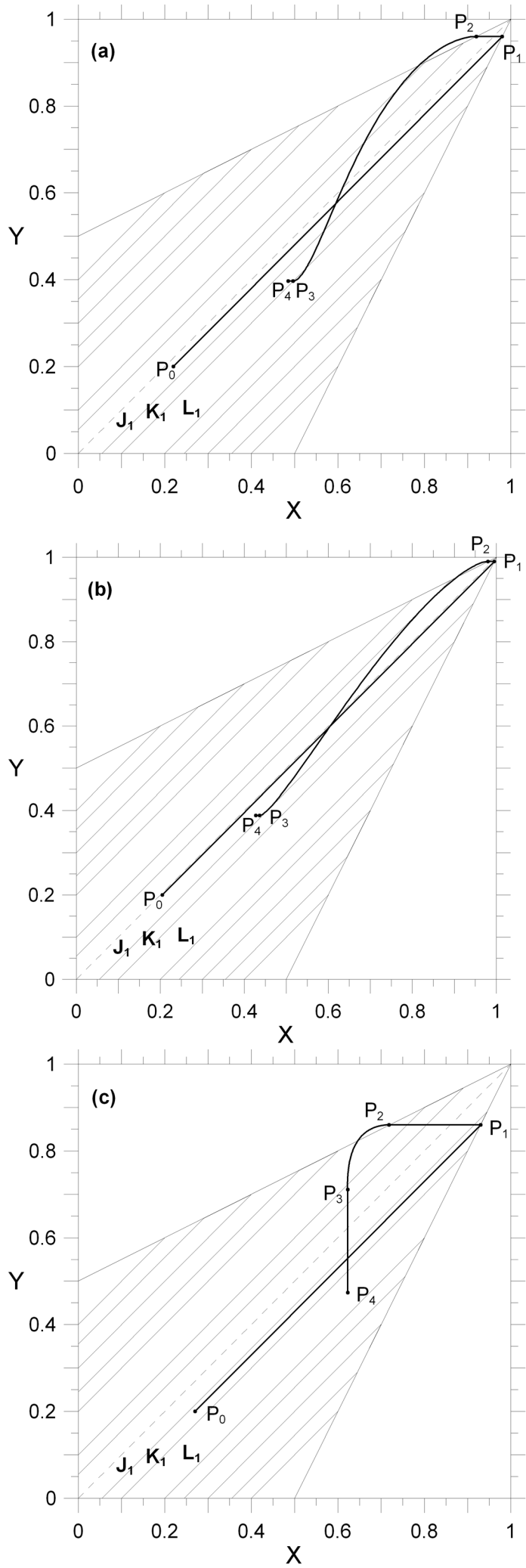

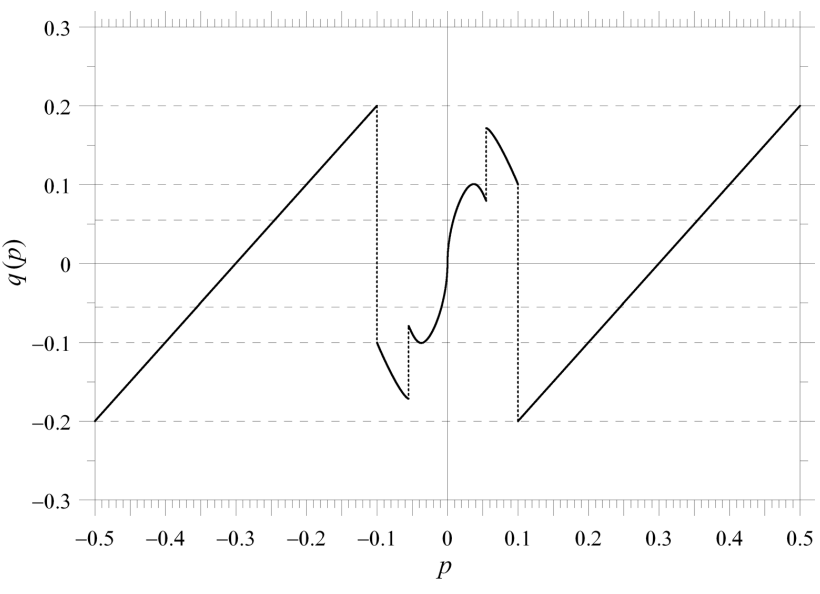

Fig. 4. Function $q(p)$ giving the position of the system in $Q$ after a slip event $(\epsilon=0.7, \alpha=1)$.

Case $P_{0} \in S_{1}$. This case is present only when $a+U<A$. Using Eqs. (9) and (14), we find that $S_{1}$ exists only if $\epsilon$ is greater than

$\epsilon_{0}=\frac{1+4 \alpha}{2(1+3 \alpha)}$

As $\alpha$ varies between 0 and $\infty$, we have $1 / 2 \leq \epsilon_{0}<2 / 3$. Hence $S_{1}$ is always present if $\epsilon>2 / 3$. It is easy to see that, if $P_{0} \in S_{1}$, then $P_{2} \in L_{1}$ whence the system enters a limit cycle.

In summary, the position of the representative point of the system in the phase space after a slip event can be expressed as

$q= \begin{cases}Y_{2}-X_{2}, & p=0 \\ Y_{4}-X_{4}, & 0<|p|<a \\ Y_{2}-X_{2}, & a<|p| \leq A\end{cases}$

In terms of $p$,

$q(p)= \begin{cases}p, & p=0 \\ f(p), & 0<|p|<a \\ p \pm U, & \mp a<p \leq \mp A\end{cases}$

where $f(p)$ is calculated numerically from the Appendix A. The function $q(p)$ is shown in Fig. 4 in the case $\alpha=1$ and $\epsilon=0.7$, entailing $c \simeq 0.055, a=0.1, b=0.2, U=0.3, A=$ 0.5 . Subset $S_{1}$ is present, since $\epsilon_{0}=5 / 8$. The function is discontinuous at $p= \pm a$ and $\pm c$.

Figure 5 shows a graph of $a, b, U$ and $A$ as functions of $\alpha$, in the case $\epsilon=0.7$. The distance between the curves shows how the thicknesses of the different subsets change as functions of $\alpha$. It is evident that a given point $P_{0}$ may belong to different subsets of $Q$ according to the value of $\alpha$.

Fig. 3. Orbits of the system in the cases (a) $p=-0.02$, (b) $p=$ -0.005 and (c) $p=-0.07(\epsilon=0.7, \alpha=1)$. 


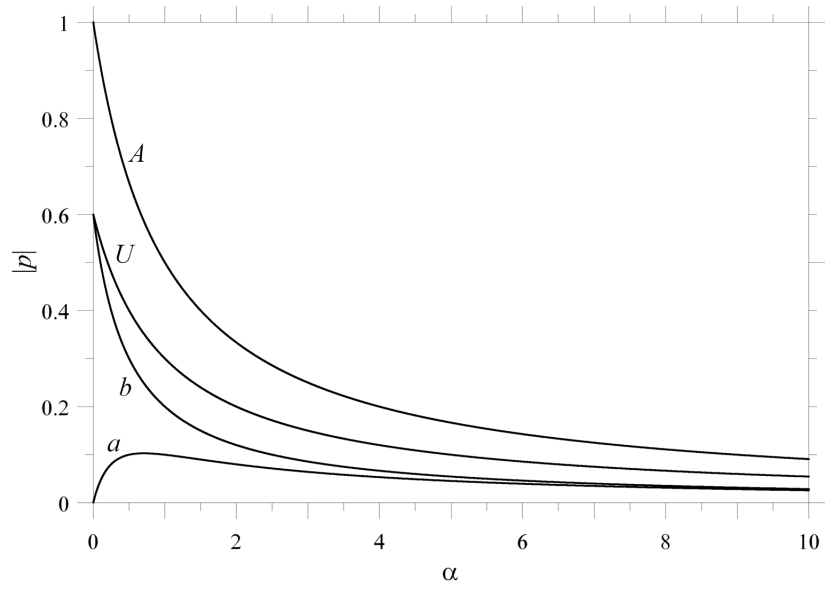

Fig. 5. The values $a, b, U$ and $A$ of $|p|$ as functions of the coupling degree $\alpha(\epsilon=0.7)$.

\section{Force distribution}

The evolution of the system is controlled by the value of $p$, expressing the difference of forces exerted on the blocks according to the equation (Dragoni and Santini, 2010)

$\Delta F=(1+2 \alpha) p$

Its magnitude $|\Delta F|$ is plotted in Fig. 6 as a function of $\alpha$. Curves in this graph are obtained from Eq. (47), with $p$ equal to $a, b, U, U+a$ and $A$ respectively. The graph shows how the position of the representative point in $Q$ is related to the distribution of forces on blocks. For instance, if $\alpha=1$, the admissible force difference for a limit cycle ranges from 30 to $60 \%$ of static friction. The interval shrinks rapidly as $\alpha$ increases: in the case of strong coupling, $|\Delta F|$ must be close to $2(1-\epsilon)$.

In all cases, the long-term destination of the system is one of the infinite limit cycles described in Dragoni and Santini (2010). Such cycles represent the alternate motion of the two blocks and are reached when the system enters the subset $L$. This implies that the force difference has values in a particular range, given by Eq. (47) with $a<|p|<b$.

If $|p|$ is smaller $(0<|p|<a),|\Delta F|$ is small as well. In this case, the system experiences a simultaneous motion of blocks which shifts the representative point to a different subset of $Q$, characterized by larger values of $p$, from which it can usually reach a limit cycle. However, if $|p|$ is very small, two such events are necessary. If $|p|$ is large $(b<|p|<U+a)$ so that $|\Delta F|$ is large, the system must go through a state in which $|\Delta F|$ is small before it can reach $L$. But if $|p|>U+a$ the system reaches $L$ by the motion of a single block.

The discontinuities in the function $q(p)$ at $p= \pm a$ and $\pm c$ indicate that, in the proximity of these points, small differences in the force distribution may entail very different evolutions of the system. The force distribution on blocks

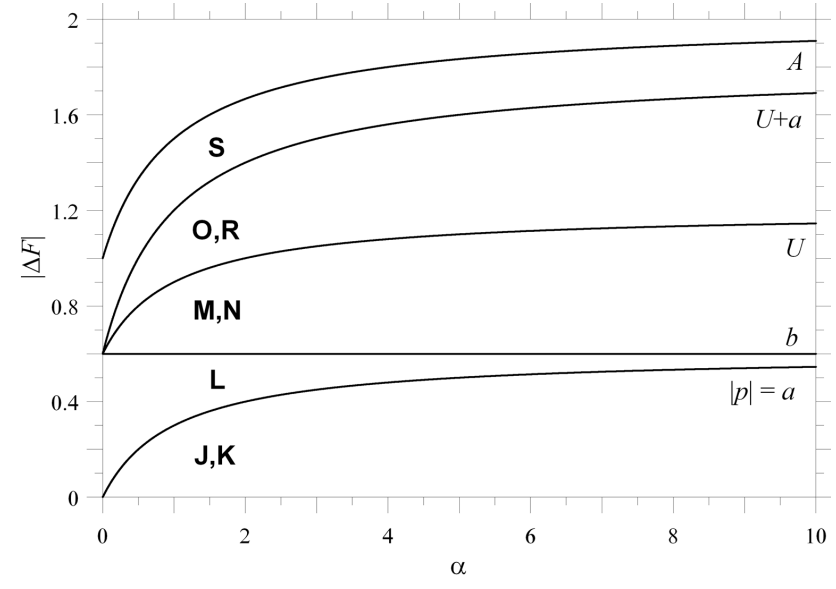

Fig. 6. The force difference $\Delta F$ as a function of $\alpha(\epsilon=0.7)$.

simulates the shear stress distribution on the equivalent fault, implying that slightly different stress distributions may result in completely different seismic sequences.

If we admit that the system is subject to force perturbations, the states of the system are unstable in the proximity of those points. In particular, the system can be driven out of $L$. This is easier as the degree of coupling $\alpha$ is larger, since the interval $[a, b]$ becomes narrower. The system will come back to $L$ only after a simultaneous motion of the blocks.

\section{Seismic moment release}

If we suppose that the displacement of a block corresponds to the slip of a fault asperity, we can calculate the associated seismic moment release $M$. Dragoni and Santini (2010) showed that each limit cycle is characterized by two seismic events separated by interseismic periods of variable duration. Each seismic event releases a moment $M_{0}$, corresponding to an amount $U$ of slip of a single asperity. In the particular case when one interseismic period is equal to zero, we may assert that a single event with moment release $2 M_{0}$ takes place in the cycle. In fact this is generated by two separate slip events taking place with zero delay: there is no simultaneous slip of the two asperities.

We now consider the simultaneous slip of the two asperities occurring when $P_{0}$ is in region $J_{1}$ or $K_{1}$. When the system is at $P_{4}$, the displacements of blocks 1 e 2 are respectively

$U_{1}=X_{1}-X_{4}, \quad U_{2}=Y_{1}-Y_{4}$

where the coordinates of $P_{4}$ are calculated in the Appendix A. The total seismic moment is then

$M=M_{0} \frac{U_{1}+U_{2}}{U}$ 


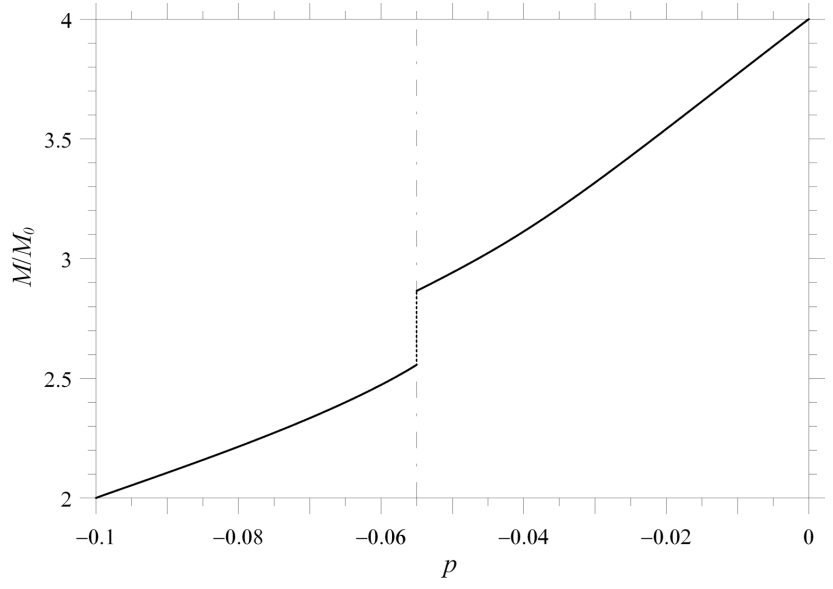

Fig. 7. Seismic moment release associated with simultaneous asperity failure as a function of $p(\epsilon=0.7, \alpha=1)$. The discontinuity is at $p=-c$.

Figure 7 shows a graph of $M$ as a function of $p$, with $-a \leq$ $p \leq 0$. The moment can be calculated analytically at the end points of this interval. If $p=-a$, we have

$U_{1}=U_{2}=U$

and

$M=2 M_{0}$

For lower values of $|p|, U_{1}$ and $U_{2}$ become greater than $U$ and $M$ is an increasing function of $p$, with a discontinuity at $p=-c$. The discontinuity is a consequence of the homologous discontinuity in the fuction $q(p)$ and means that, in the proximity of $p=-c$, a small difference in stress may entail a remarkable difference in seismic moment. When $p=0$, Eqs. (42) and (43) give

$U_{1}=U_{2}=2(1-\epsilon)$

and

$M=2(1+\alpha) M_{0}$

which is the maximum value of seismic moment. Therefore the moment release associated with the simultaneous failure of the two asperities is always greater than the maximum value, equal to $2 M_{0}$, that can be released in a limit cycle. According to Eq. (53), $M$ depends on the degree of coupling $\alpha$. For any value of $\alpha>0, M$ is not simply the sum of moments $M_{0}$ released by the two asperities when they slip separately. This indicates that the asperities interact during the slip event, with a seismic moment increasing linearly with $\alpha$. If $\alpha=1$, the maximum value is as large as $4 M_{0}$. The earthquakes generated by the simultaneous failures of the two asperities can be considered as the largest earthquakes that can be generated by the fault.

\section{Conclusions}

The model is a greatly simplified picture of a seismic source, but may shed some light on the mechanics of a fault system made of two asperities. Due to the many simplifying assumptions (in particular that of equal asperity strengths), the model is not intended to simulate the behaviour of any real fault, but to highlight the mechanisms that are responsible for the aperiodicity of the seismic activity observed in fault systems.

We have proven that, whichever the initial state is, the long-term behaviour of the system is a limit cycle with a particular recurrence pattern of earthquakes. The limit cycle requires a certain stress distribution repeating periodically. If an external stress transfer alters such a distribution, the fault may experience a larger earthquake, with the simultaneous failure of the two asperities, which finally restores a stress distribution compatible with periodic behaviour.

The fact that the phase space can be divided into narrow stripes corresponding to very different evolutions of the system implies that the system is sensitive to small perturbations. Depending on the value of the coupling between asperities, a stress perturbation in the order of $10 \%$ of static friction or less can be sufficient to move the system to a different subset of the phase space. This has relevant implications for a fault, which can not be considered an isolated system, but is subject to stress transfers from earthquakes generated by neigbouring faults.

Under the model assumptions, stress transfers from neigbouring faults have a key role in the mechanics of the fault system. The system tends to a periodic behaviour, but its orbit is frequently modified by external perturbations, which have usually recurrence times shorter than those of earthquakes generated by the fault in the periodic regime. In many cases the perturbation will shift the orbit to a different limit cycle with a different recurrence pattern, but equal seismic moments. However, when the system is taken out of the range of the admissible stress distributions for limit cycles, an earthquake is generated with a seismic moment that can be several times larger. This is an effect of the interaction between the asperities: the seismic moment is greater than the sum of the moments that the two asperities release when they slip separately.

\section{Appendix A}

We calculate here the orbits in the cases $P_{0} \in J_{1}$ and $P_{0} \in K_{1}$. In both cases, the first segment of the orbit belongs to line Eq. (10), which intercepts line 1 at point $P_{1}$ with coordinates

$X_{1}=1+\alpha p, \quad Y_{1}=1+\omega^{2} p$

The second segment belongs to line $Y=Y_{1}$. It is given by Eq. (17) with initial conditions

$\bar{X}=X_{1}, \quad \bar{V}=0$ 
and the substitution $\bar{Y}=Y_{1}$. Then

$X(T)=X_{1}-\frac{U}{2}(1-\cos \omega T)$

The orbit intercepts line 2 at point $P_{2}$ with coordinates

$X_{2}=1+\frac{\omega^{4}}{\alpha} p, \quad Y_{2}=1+\omega^{2} p$

From Eq. (A3) with $X=X_{2}$, we obtain the time $T_{12}$ taken by the block to move from $P_{1}$ to $P_{2}$ :

$T_{12}=\frac{1}{\omega} \arccos \left(1+\frac{2 p}{a}\right)$

The velocity of block 1 at $T=T_{12}$ is then

$V_{2}=-\frac{U}{2} \omega \sin \omega T_{12}$

or, thanks to (A5),

$V_{2}=-\frac{\omega \Omega^{2}}{\alpha} \sqrt{-p(a+p)}$

The third segment is curvilinear and has the parametric Eqs. (24) and (25) with

$\bar{X}=X_{2}, \quad \bar{Y}=Y_{2}, \quad \bar{V}=V_{2}$

The segment terminates at point $P_{3}$ where one of the blocks stops. The shape of the segment depends on the value of $p$, which determines which block stops first. In order to calculate the coordinates of $P_{3}$, we solve Eqs. (28) and (29) and obtain the duration $T_{23}$ of simultaneous motion according to Eq. (30). It results $T_{23}=T_{y}$ if $-c<p<0\left(P_{0} \in J_{1}\right)$; $T_{23}=T_{x}$ if $-a<p \leq-c\left(P_{0} \in K_{1}\right)$. The value of $c$ is defined by the condition that $\dot{X}(T)$ has a minimum equal to zero at $T=T_{x}$. The orbits are therefore different in the two cases.

\section{A1 Case $P_{0} \in J_{1}$}

In this case block 2 stops first. The coordinates of $P_{3}$ are calculated from (24) and (25) as

$X_{3}=X\left(T_{y}\right), \quad Y_{3}=Y\left(T_{y}\right)$

The velocity of block 1 at $P_{3}$ is

$V_{3}=\dot{X}\left(T_{y}\right)$

or, thanks to (29),

$V_{3}=\left(2 \epsilon-X_{2}-Y_{2}\right) \sin T_{y}+V_{2} \cos T_{y}$

The fourth segment of the orbit belongs to line $Y=Y_{3}$, representing the motion of block 1 . The solution is given by (17) with

$\bar{X}=X_{3}, \quad \bar{Y}=Y_{3}, \quad \bar{V}=V_{3}$
The segment terminates at point $P_{4}=\left(X_{4}, Y_{4}\right)$. From (19), block 1 stops after a time

$T_{34}=\frac{1}{\omega} \arctan \frac{\omega V_{3}}{\omega^{2} X_{3}-\epsilon-\alpha Y_{3}}$

From (17),

$$
\begin{aligned}
X\left(T_{34}\right)= & \frac{\epsilon+\alpha Y_{3}}{\omega^{2}}+\left(X_{3}-\frac{\epsilon+\alpha Y_{3}}{\omega^{2}}\right) \cos \omega T_{34} \\
& +\frac{V_{3}}{\omega} \sin \omega T_{34}
\end{aligned}
$$

or, thanks to (A13),

$$
X\left(T_{34}\right)=\frac{1}{\omega^{2}}\left[\epsilon+\alpha Y_{3}-\sqrt{\left(\omega^{2} X_{3}-\epsilon-\alpha Y_{3}\right)^{2}+\omega^{2} V_{3}^{2}}\right]
$$

Then

$$
X_{4}=X\left(T_{34}\right), \quad Y_{4}=Y_{3}
$$

\section{A2 Case $P_{0} \in K_{1}$}

In this case block 1 stops first. The coordinates of $P_{3}$ are calculated from (24) and (25) as

$X_{3}=X\left(T_{x}\right), \quad Y_{3}=Y\left(T_{x}\right)$

The velocity of block 2 at $P_{3}$ is

$V_{3}=\dot{Y}\left(T_{x}\right)$

or, thanks to (28),

$V_{3}=\left(2 \epsilon-X_{2}-Y_{2}\right) \sin T_{x}+V_{2} \cos T_{x}$

The fourth segment of the orbit belongs to line $X=X_{3}$, representing the motion of block 2 . The solution is given by (21) with

$\bar{X}=X_{3}, \quad \bar{Y}=Y_{3}, \quad \bar{V}=V_{3}$

The segment terminates at point $P_{4}=\left(X_{4}, Y_{4}\right)$. From (22), block 2 stops after a time

$T_{34}=\frac{1}{\omega} \arctan \frac{\omega V_{3}}{\omega^{2} Y_{3}-\epsilon-\alpha X_{3}}$

Then

$Y\left(T_{34}\right)=\frac{1}{\omega^{2}}\left[\epsilon+\alpha X_{3}-\sqrt{\left(\omega^{2} Y_{3}-\epsilon-\alpha X_{3}\right)^{2}+\omega^{2} V_{3}^{2}}\right]$

and

$X_{4}=X_{3}, \quad Y_{4}=Y\left(T_{34}\right)$

Acknowledgements. We thank the editor Ilya Zaliapin, Rodolfo Console and an anonymous referee for useful comments and suggestions on the paper.

Edited by: I. Zaliapin

Reviewed by: R. Console and another anonymous referee 


\section{References}

Belardinelli, M. E. and Belardinelli, E.: The quasi-static approximation of the spring-slider motion, Nonlin. Processes Geophys., 3, 143-149, doi:10.5194/npg-3-143-1996, 1996.

Burridge, R. and Knopoff, L.: Model and theoretical seismology, Bull. Seismol. Soc. Am., 57, 341-371, 1967.

Byerlee, J.: Friction of rocks, Pure Appl. Geophys., 116, 616-626, 1978.

Cao, T. and Aki, K.: Seismicity simulation with a mass-spring model and a displacement hardening-softening friction law, Pure Appl. Geophys., 122, 10-23, 1984.

Cao, T. and Aki, K.: Seismicity simulation with a rate and state dependent friction law, Pure Appl. Geophys., 124, 487-513, 1986.

Carlson, J. and Langer J.: Mechanical model of an earthquake fault, Phys. Rev. A, 40, 6470-6484, 1989.

Carlson, J. and Langer, J.: Properties of earthquakes generated by fault dynamics, Phys. Rev. Lett., 62, 2632-2635, 1989 b.

Carlson, J., Langer, J., and Shaw, B.: Dynamics of earthquake fault, Rev. Mod. Phys., 66, 657-659, 1994.

Cohen, S.: Computer simulation of earthquakes, J. Geophys. Res., 82, 3781-3796, 1977.

de Sousa Vieira, M.: Chaos in a simple spring-block system, Phys. Lett. A, 198, 407-414, 1995.

di Bernardo, M., Budd, C., Champneys, A. R., and Kowalczyk, P.: Piecewise-smooth Dynamical Systems, 483 pp., Springer, 2008.

Dieterich, J. H.: Time dependent friction as a possible mechanism for aftershocks, J. Geophys. Res., 77, 3771-3781, 1972.

Dieterich, J. H.: Constitutive properties of faults with simulated gouge, in Mechanical Behavior of Crustal Rocks, edited by: Carter, N. L., Friedman, M., Logan, J. M., and Stearns, D. W., Am. Geophys. Union, Geophys. Monogr., 24, 103-120, 1981.

Dragoni, M. and Santini, S.: Simulation of the long-term behaviour of a fault with two asperities, Nonlin. Processes Geophys., 17, 777-784, doi:10.5194/npg-17-777-2010, 2010.

Erickson, B., Birnir, B., and Lavalle, D.: A model for aperiodicity in earthquakes, Nonlin. Processes Geophys., 15, 1-12, doi:10.5194/npg-15-1-2008, 2008.
Galvanetto, U.: Sliding bifurcations in the dynamics of mechanical systems with dry friction - remarks for engineers and applied scientists, J. Sound Vibration, 276, 121-139, 2004.

Gu, J. C., Rice, J. R., Ruina, A. L., and Tse, S. T.: Slip motion and stability of a single degree of freedom elastic system with rate and state dependent friction, J. Mech. Phys. Sol., 32, 167-196, 1984.

Gu, J. C. and Wong T. F.: Effects of loading velocity, stiffness and inertia on the dynamics of a single degree of freedom springslider system, J. Geophys. Res., 96, 21677-21691, 1991.

$\mathrm{He}, \mathrm{C} .:$ Interaction between two sliders in a system with rate- and state-dependent friction, Science in China, Series D, 46, 67-74, 2003.

Huang, J. and Turcotte, D. L.: Are earthquakes an example of deterministic chaos?, Geophys. Res. Lett., 17, 223-226, 1990a.

Huang, J. and Turcotte, D. L.: Evidence for chaotic fault interactions in the seismicity of the San Andreas fault and Nankai trough, Nature, 348, 234-236, 1990b.

Huang, J. and Turcotte, D. L.: Chaotic seismic faulting with massspring model and velocity-weakening friction, Pure Appl. Geophys., 138, 569-589, 1992.

McCloskey, J. and Bean, C. J.: Time and magnitude predictions in shocks due to chaotic fault interactions, Geophys. Res. Lett., 19, 119-122, 1992.

Nussbaum, J. and Ruina, A.: A two degree-of-freedom earthquake model with static/dynamic friction, Pure Appl. Geophys., 125, 629-656, 1987.

Rice, J. R. and Tse, S. T.: Dynamic motion of a single degree of freedom system following a rate and state dependent friction law, J. Geophys. Res., 91, 521-530, 1986.

Ruina, A.: Slip instability and state variable friction laws, J. Geophys. Res., 88, 10359-10370, 1983.

Rundle, J. B. and Jackson, D. D.: Numerical simulation of earthquake sequences, Bull. Seismol. Soc. Am., 67, 1363-1377, 1977.

Turcotte, D. L.: Fractals and Chaos in Geology and Geophysics, 2nd Edn., Cambridge University Press, Cambridge, 1997. 